\title{
A comparison of timed artificial insemination and automated activity monitoring with hormone intervention in 3 commercial dairy herds
}

\author{
K. A. Dolecheck, ${ }^{*}$ W. J. Silvia, ${ }^{* 1}$ G. Heersche Jr., ${ }^{*}$ C. L. Wood,† K. J. McQuerry,† and J. M. Bewley* \\ *Department of Animal and Food Sciences, and \\ †Department of Statistics, University of Kentucky, Lexington 40546
}

\begin{abstract}
The objective of this study was to compare the reproductive performance of cows inseminated based on automated activity monitoring with hormone intervention (AAM) to cows from the same herds inseminated using only an intensive timed artificial insemination (TAI) program. Cows $(\mathrm{n}=523)$ from 3 commercial dairy herds participated in this study. To be considered eligible for participation, cows must have been classified with a body condition score of at least 2.50, but no more than 3.50, passed a reproductive tract examination, and experienced no incidences of clinical, recorded metabolic diseases in the current lactation. Within each herd, cows were balanced for parity and predicted milk yield, then randomly assigned to 1 of 2 treatments: TAI or AAM. Cows assigned to the TAI group were subjected to an ovulation synchronization protocol consisting of presynchronization, Ovsynch, and Resynch for up to 3 inseminations. Cows assigned to the AAM treatment were fitted with a leg-mounted accelerometer (AfiAct Pedometer Plus, Afimilk, Kibbutz Afikim, Israel) at least $10 \mathrm{~d}$ before the end of the herd voluntary waiting period (VWP). Cows in the AAM treatment were inseminated at times indicated by the automated alert system for up to $90 \mathrm{~d}$ after the VWP. If an open cow experienced no AAM alert for a $39 \pm 7$-d period (beginning at the end of the VWP), hormone intervention in the form of a single injection of either $\mathrm{PGF}_{2 \alpha}$ or GnRH (no TAI) was permitted as directed by the herd veterinarian. Subsequent to hormone intervention, cows were inseminated when alerted in estrus by the AAM system. Pregnancy was diagnosed by ultrasound 33 to $46 \mathrm{~d}$ after insemination. Pregnancy loss was determined via a second ultrasound after $60 \mathrm{~d}$ pregnant. Timed artificial insemination cows experienced a median 11.0 d shorter time to first service. Automated activity-
\end{abstract}

Received June 6, 2015.

Accepted October 26, 2015.

${ }^{1}$ Deceased.

${ }^{2}$ Corresponding author: jbewley@uky.edu monitored cows experienced a median 17.5-d shorter service interval. No treatment difference in probability of pregnancy to first AI, probability of pregnancy to repeat AI, pregnancy loss, time to pregnancy, or proportion of pregnant cows at $90 \mathrm{~d}$ past the VWP existed. Based on these results, inseminating cows using AAM with hormone intervention can achieve a level of reproductive performance comparable to TAI. Considering the strict cow selection criteria used in this study, interpretation of results for on-farm implementation should be performed cautiously; the results cannot be directly extrapolated to whole herds of cows.

Key words: automated estrus detection, automated activity monitoring, timed artificial insemination, reproductive management

\section{INTRODUCTION}

Estrus detection efficiency is a major limiting factor in dairy reproductive performance (Heersche and Nebel, 1994; Nebel and Jobst, 1998; Ferguson and Skidmore, 2013). The Dairy Records Management Systems reported mean yearly estrus detection rate on US Holstein herds (including all reproductive-management programs) as $44.9 \%$ in 2014 (DRMS, 2015). Poor estrus detection may be a result of the short duration of estrus $(6.2 \pm 0.5 \mathrm{~h})$ observed in high-producing North American Holstein cows (Lopez et al., 2004). Increasing age and environmental factors (greater ambient temperature, uncomfortable housing, and so on) can also negatively affect the length and intensity of estrus expression (Vailes and Britt, 1990; López-Gatius et al., 2005; Palmer et al., 2010). Timed artificial insemination (TAI), commonly accomplished through a synchronization protocol known as Ovsynch (Pursley et al., 1995), has successfully been implemented on many farms as an aid or replacement for visual estrus detection. Unfortunately, a lack of protocol compliance (i.e., missed injections) often reduces the success of TAI (Galvão et al., 2013). Additionally, consumer concerns surrounding hormone use could limit this management option in the future (Saint-Dizier and Chastant-Maillard, 2012; Nebel, 2013). 
One alternative to TAI is automated estrus detection (AED) technologies. Parameters with potential for AED include mounting events, activity level, lying time, rumination events, blood or milk progesterone levels, feeding time, and body temperature (Senger, 1994; Saint-Dizier and Chastant-Maillard, 2012; Fricke et al., 2014a). Challenges associated with AED include determining how and when to intervene with anovular or otherwise undetected cows and determining the best time to inseminate relative to behavioral changes (Fricke et al., 2014a). Variation among animals complicates both of these factors. Another drawback associated with AED, as identified in producer surveys, is the large investment cost and uncertainty in payback period (Russell and Bewley, 2013; Borchers and Bewley, 2015).

Most AED technologies currently available to dairy farmers work via automated activity monitoring (AAM). In an evaluation by Liu and Spahr (1993), $74.2 \%$ of estrus events were detected by AAM when using pregnancy diagnosis to define estrus periods. In comparison, direct visual observation only detected $57.6 \%$ of estrus events. Cavalieri et al. (2003) compared AAM to a rump-mounted pressure-sensitive mounting detector and tail paint using milk progesterone levels and pregnancy diagnosis as verification. They found no differences in the proportion of true estrus events detected (81.4, 88.4, and 91.3\%, respectively). Estrus detection risk of AAM, as reported in other studies, ranges between 51.0 and $89.2 \%$ in both confinement and pasture situations (Roelofs et al., 2005; Dela Rue et al., 2014).

Direct comparisons of reproductive performance in dairy cows inseminated by TAI versus AAM are limited. Galon (2010) found no difference in first service conception risk (CR; 17.6 vs. $22.6 \%$, respectively) between cows inseminated based on AAM versus those on a TAI protocol, but a small sample size $(\mathrm{n}=140)$ from only 1 herd limits the interpretation of those results. Neves et al. (2012) compared TAI to AAM using over 900 animals from 3 herds, finding no differences in pregnancy risk (15.9 vs. $14.6 \%)$ or CR (30.0 vs. $31.0 \%$ ). However, results from that study are difficult to interpret because insemination based on visual detection was permitted in both treatment groups and differing TAI protocols were used on each herd. In a follow-up study, Neves and LeBlanc (2015) surveyed 35 farms using AAM and 53 farms using TAI in Canada, finding no difference in 21 -d pregnancy risk ( $18.0 \%$ vs. $17.3 \%)$, CR (39.4\% vs. $38.1 \%$ ), or 21 -d insemination risk $(49.7 \%$ vs. $49.0 \%)$. Limitations also existed in that study, including that comparisons were observational only and made between and not within herds.

The objective of the current study, using 3 commercial dairy herds, was to compare the reproductive performance of cows inseminated based on AAM with hormone intervention to cows from the same herds inseminated using only an intensive TAI program. Both reproductive-management programs tested represent potential alternatives to traditional visual estrus detection. To provide a controlled experimental environment, as free as possible from confounding factors, only cows meeting specific criteria were included in the comparison.

\section{MATERIALS AND METHODS}

Our study was conducted in 3 commercial dairy herds in Kentucky. All 3 herds milked Holsteins exclusively. Farm location, herd size, and willingness to participate were considered during herd selection. The size, housing type, and general performance characteristics of each herd are presented in Table 1.

Table 1. Demographic and management information from 3 dairy herds used in a study (beginning October 2012) comparing timed AI and automatic activity monitoring with hormone intervention as reproductivemanagement programs

\begin{tabular}{|c|c|c|c|}
\hline Item & Herd A & Herd B & Herd C \\
\hline Lactating herd size $\mathrm{e}^{1}(\mathrm{n})$ & 261 & 190 & 432 \\
\hline Breed & Holstein & Holstein & Holstein \\
\hline Milking frequency per day & 2 & 2 to $3^{2}$ & 3 \\
\hline Rolling herd mean milk production ${ }^{1}(\mathrm{~kg})$ & 12,230 & 11,040 & 9,381 \\
\hline Voluntary waiting period (DIM) & 80 & 60 & 60 \\
\hline Median BCS at study enrollment ${ }^{3}$ & 2.75 & 3.00 & 3.00 \\
\hline Housing type & Freestall and $\mathrm{CBPB}^{4}$ & Freestall & Freestall \\
\hline Concrete & Grooved & Ungrooved & Grooved \\
\hline
\end{tabular}

${ }^{1}$ Size at the conclusion of the study; herd A and B: June 2014; herd C: February 2014.

${ }^{2}$ Herd B changed from 2 to 3 times daily milking in December 2013.

${ }^{3}$ As evaluated using the 1 to 5, 0.25-point BCS system developed by Ferguson et al. (1994).

${ }^{4} \mathrm{CBPB}=$ compost-bedded pack barn. Cows in the study may have been housed on 1 or switched between these

2 housing systems in herd A. 


\section{Study Design}

Each herd manager agreed to enroll cows in the study for $1 \mathrm{yr}$, beginning in October 2012. At the conclusion of $1 \mathrm{yr}$, cows from herds $\mathrm{A}$ and $\mathrm{B}$ continued to be enrolled for an additional 3 mo. All cows in herd C completed the study by January 2014. The last cows in herds A and B completed the study in June 2014.

Cows were assigned to a treatment group in 14-d intervals when they reached 17 to $31 \mathrm{~d}$ before the end of the voluntary waiting period (VWP), which differed by herd (Table 1). To be eligible for this experiment, cows had to meet specific health requirements. First, each cow underwent a routine reproductive exam by the herd veterinarian to verify normal progression of uterine involution (free of any clinical signs of metritis or pyometra) and expected ovarian activity (determined by palpating the ovaries for corpus luteum and preovulatory follicle presence). Cows were excluded from the study if they did not pass this exam ( $\mathrm{n}=$ 149). Second, each cow's BCS was evaluated using a 1- to 5-scale (Ferguson et al., 1994). Cows with a BCS less than 2.50 (emaciated; $\mathrm{n}=27$ ) or greater than 3.50 (fat; $\mathrm{n}=20$ ) were excluded from the study. Remaining cows were grouped into 2 BCS categories: 2.50 to 2.75 and 3.00 to 3.50. Third, cows must have been free of clinical metabolic disorders postcalving. Cows were excluded from the study if they had a record of experiencing a clinical metabolic disorders in the current lactation $(\mathrm{n}=11)$. These criteria were set in an attempt to reduce the effect of factors known to influence cow fertility, allowing a controlled comparison of the 2 nonvisual reproductive-management programs. One factor that may have affected fertility that was not assessed is lameness. Because our study was conducted on commercial farms, collecting records on lameness occurrence was difficult. If left to the herd managers, timing of lameness identification would likely have been inconsistent. On-farm assessment could have been conducted by the researchers, but identifying precise lameness onset would have required more frequent visits to each herd.

Eligible cows were classified by parity (primiparous or multiparous) and predicted milk yield using DHIA records. Each cow's predicted milk yield was classified as greater or less than herd PTA milk mean. If PTA milk was not available for a cow, estimated relative producing ability milk was used. If neither PTA milk nor estimated relative producing ability milk was available, first test day milk production was used. Assignment of cows to treatments (TAI or AAM) followed, alternating alike herdmates (determined by parity category and predicted milk yield classification) between treatments. This allocation procedure resulted in treatments bal- anced for parity $(1.94 \pm 1.13$ and $2.13 \pm 1.35$ for TAI and AAM, respectively) and summit milk, defined as the mean of the 2 greatest milk yields from the first 3 test days $(39.62 \pm 8.61$ and $39.43 \pm 8.56 \mathrm{~kg}$ for TAI and AAM, respectively). Forty-five cows were enrolled twice during the study period. Because this number was too small to correctly account for repeated measures, only the first completed enrollment for each cow was included in the statistical analysis.

Cows were managed according to the experimental protocol until $90 \mathrm{~d}$ after the end of the VWP. At that point, the herd manager was permitted to use any reproductive management practice he or she desired to breed nonpregnant cows.

\section{TAI Treatment}

Ovulation synchronization in cows assigned to the TAI treatment involved a combination of protocols commonly referred to as G7G (defined below), Ovsynch, and Resynch (Figure 1). The G7G presynchronization began 6 to $19 \mathrm{~d}$ before the end of the VWP, with groups starting every $14 \mathrm{~d}$. The G7G protocol consisted of $\mathrm{PGF}_{2 \alpha}$ (dinoprost tromethamine, Lutalyse; Zoetis, Florham Park, NJ; 25 mg i.m.) and GnRH (gonadorelin diacetate tetrahydrate, Cystorelin; Merial Limited, Duluth, GA; $100 \mu \mathrm{g}$ i.m.) administered 9 and $7 \mathrm{~d}$ before beginning Ovsynch, respectively. The Ovsynch protocol consisted of GnRH on d $0(0800 \mathrm{~h})$, followed by $\mathrm{PGF}_{2 \alpha}$ on d $7(0800 \mathrm{~h})$, GnRH on d $9(1600 \mathrm{~h})$, and insemination on $\mathrm{d} 10,0$ to $13 \mathrm{~d}$ after the end of the VWP (0800 h; Pursley et al., 1995). Resynch began with GnRH 1 wk before pregnancy diagnosis and continued only if cows were not pregnant. Resynch cows received $\mathrm{PGF}_{2 \alpha}$ at the time of nonpregnancy diagnosis, followed by GnRH and AI 56 and $72 \mathrm{~h}$ later, respectively. This method allowed up to 3 inseminations in TAI cows during the 90-d study period. Herd managers supervised the synchronization protocol and each possessed previous experience with TAI protocols.

\section{AAM Treatment}

The AAM technology, measuring $9.2 \times 7.4 \times 2.4 \mathrm{~cm}$ and weighing $92 \mathrm{~g}$ (AfiAct Pedometer Plus, Afimilk, Kibbutz Afikim, Israel), was attached to a rear leg at least $10 \mathrm{~d}$ before the end of the VWP to establish the normal activity level of each cow. In herds B and C, only cows assigned to the AAM treatment received the ankle-mounted, AAM technology. In herd A, all cows received the AAM technology, but only AAM treatment cows were inseminated using the technology alerts. The AAM system continuously recorded activity level using a 3-axis accelerometer that relayed information to 


\section{G7G-Ovsynch}

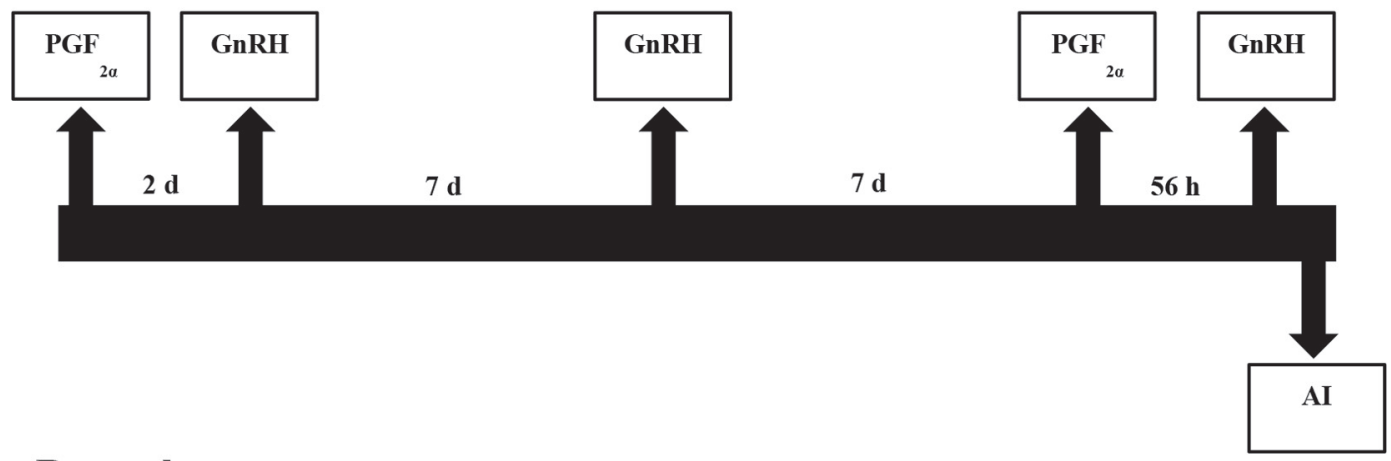

\section{Resynch}

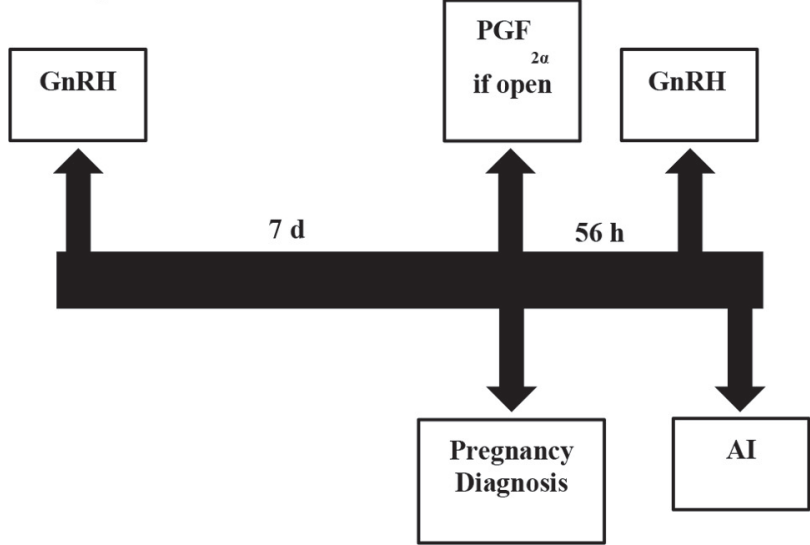

Figure 1. Timed AI G7G, Ovsynch, and Resynch protocols using PGF $_{2 \alpha}$ and GnRH. The G7G presynchronization began 6 to 19 d before the end of the voluntary waiting period, with groups starting every $14 \mathrm{~d}$. Artificial insemination occurred $16 \mathrm{~h}$ after the last GnRH injection, 0 to $13 \mathrm{~d}$ after the end of the voluntary waiting period.

a computer during milking. Steps per hour since the last milking and a 10-d backward moving mean steps per hour were automatically calculated after each data download. An estrus alert was generated when the most recent measurement of a cow's steps per hour exceeded her 10-d backward moving mean by a specific amount, as determined using a proprietary algorithm consistent across herds. Herd managers were instructed to check the AAM alert list twice per day and inseminate AAM cows once per alerted estrus, unless specific management practices (i.e., pen changes, hoof trimming, and so on) indicated a false alert. All herds began using the AAM system in March 2012 (6 mo before beginning the study) and were considered past the initial adjustment and learning stage at the beginning of the study.

If an open cow experienced no AAM alert for a period of $39 \pm 7 \mathrm{~d}$ (beginning at the end of the VWP and continuing throughout the 90-d study period), hormone intervention in the form of a single injection of either $\mathrm{PGF}_{2 \alpha}$ or GnRH (no TAI) was permitted as directed by the herd veterinarian after palpation. Subsequent to hormone intervention, cows were inseminated when alerted in estrus by the AAM system. Regardless of hormone intervention, cows remained in the AAM treatment for statistical analysis.

\section{Pregnancy Diagnosis}

The herd veterinarian or a trained researcher conducted pregnancy diagnosis via ultrasound at 39 (herd $\mathrm{B}$ and herd $\mathrm{C}$ ) or $40 \mathrm{~d}$ (herd A) postinsemination for TAI cows and $39 \pm 7$ (herd B and C) or $40 \pm 7 \mathrm{~d}$ (herd A) postinsemination for AAM cows. Pregnancy loss was determined via a second ultrasound after $60 \mathrm{~d}$ pregnant.

\section{Herd Records}

Calving dates, predicted milk yield, insemination dates, pregnancy diagnosis, and culling information for each herd were obtained through PCDART (Dairy Records Management Systems, Raleigh, NC). These records were used to calculate time to first service, probability of pregnancy to first AI, probability of preg- 
nancy to repeat AI, service interval, pregnancy loss, time to pregnancy, and the proportion of cows pregnant at $90 \mathrm{~d}$ past the VWP. Because of differences in herd VWP, time to first service and time to pregnancy was calculated as days past the VWP.

Cows in the AAM treatment that were never inseminated were excluded from the statistical analysis of probability of pregnancy to first AI, probability of pregnancy to repeat AI, service interval, and pregnancy loss. Additionally, cows that never became pregnant were excluded from the pregnancy loss statistical analysis. Table 2 includes the final number of cows from each treatment used for each analysis.

\section{Statistical Analysis}

To analyze probability of pregnancy to first AI, pregnancy loss, and proportion of cows pregnant at $90 \mathrm{~d}$ past the VWP, binomial data sets were created using 1 to identify the occurrence of each event (pregnancy or pregnancy loss) and 0 to identify no event occurrence. The GENMOD procedure of SAS 9.3 (SAS Institute Inc., Cary, NC) was used to analyze the effects of treatment, herd, parity category, BCS category, and interactions between treatment and the other covariates on the probability of pregnancy to first AI, pregnancy loss, and the proportion of cows pregnant at $90 \mathrm{~d}$ past the VWP:

$$
\begin{gathered}
y_{i j k l m}=\mu+\text { Treatment }_{i}+\text { Herd }_{j}+\text { Parity }_{k}+\text { BCS }_{l} \\
+\left(\text { Treatment }_{i} \times \text { Herd }_{j}\right)+\left(\text { Treatment }_{i} \times \text { Parity }_{k}\right) \\
+\left(\text { Treatment }_{i} \times \text { BCS }_{l}\right)+e_{i j k l m},
\end{gathered}
$$

where $y_{i j k l m}$ is the outcome variable of the $m$ th cow, in the lth BCS category, in the $k$ th parity category, in the $j$ th herd, with treatment $i ; \mu$ is the intercept; $i$ is TAI or AAM; $j$ is herd $\mathrm{A}, \mathrm{B}$, or $\mathrm{C} ; k$ is primiparous or multiparous; $l$ is BCS of 2.50 to 2.75 or 3.00 to 3.50 ; and $e_{i j k l m}$ is residual error.

A similar binomial model evaluated the probability of pregnancy to repeat AI. The only difference in that model was that a repeated measures statement was included to account for cows contributing multiple insemination events. All main effects remained in each GENMOD model, regardless of significance. Two-way interactions remained in models when $P<0.05$, as determined through stepwise backward elimination.

Survival analysis was used to evaluate the effect of treatment on time to first service (censored at the end of the study period), service interval, and time to pregnancy (censored at the end of the study period) using the PHREG procedure of SAS. The hazard ratio and $95 \%$ confidence intervals were reported from each anal-

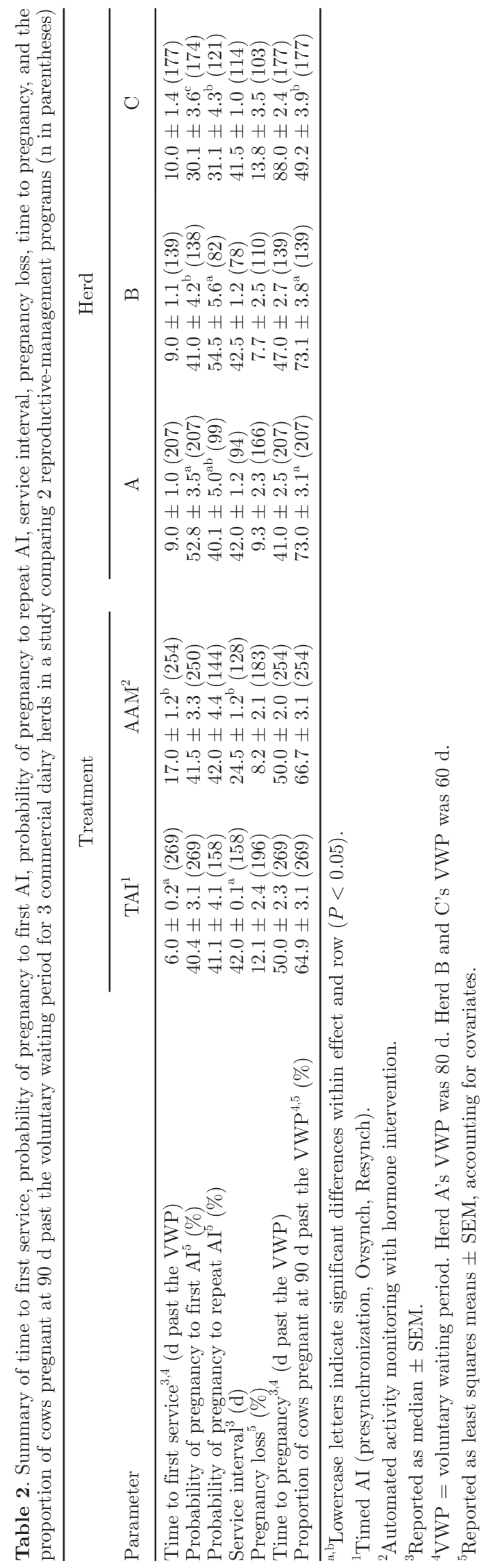


ysis. The UNIVARIATE procedure of SAS was used to calculate the median time to each event $( \pm$ SEM) for both treatments.

All analyses were designed to test for noninferiority of the AAM treatment compared with the TAI treatment. A poststudy power test was calculated and final sample size $(\mathrm{n}=523)$ was determined adequate for detecting a 5 -d difference in time to first service, service interval, and time to pregnancy between treatments as well as a $10 \%$ difference in probability of pregnancy to first $\mathrm{AI}$ and proportion of cows pregnant at $90 \mathrm{~d}$ past the VWP. An additional 114 cows per treatment would be required to find a $10 \%$ difference in probability of pregnancy to repeat AI and pregnancy loss. The final power for those analyses (the probability that the test correctly rejected the null hypothesis) were 0.61 and 0.62 , respectively. Results should be interpreted accordingly, keeping in mind the allowable difference for all analyses and the power level for the probability of pregnancy to repeat AI and pregnancy loss analyses.

\section{RESULTS AND DISCUSSION}

Throughout the study, 798 cows were initially enrolled (249 from herd A, 171 from herd B, and 378 from herd C). The study objective was to compare reproductive performance in cows inseminated based on either of the 2 nonvisual reproductive management programs. Consequently, cows reported by herd managers as being inseminated based on visual observation of estrus during the study period were removed from statistical analysis ( $\mathrm{n}=53$ ). Additionally, any cows that were (1) reported by the herd manager as having missed TAI injections $(\mathrm{n}=59),(2)$ found to be eligible for insemination during a period of AAM system failure (n $=43),(3)$ not inseminated at the time of an AAM alert as reported by the herd manager $(\mathrm{n}=6),(4)$ culled during the $90-\mathrm{d}$ study period $(\mathrm{n}=61)$, or $(5)$ removed from the study by the herd manager for health or other reasons $(\mathrm{n}=51)$ were also excluded from the statistical analysis. After removing those cows, 523 cows remained in the analysis (207 from herd A, 139 from herd B, and 177 from herd C).

Of the cows that completed the study, $51.4 \%$ were in the TAI group $(\mathrm{n}=269)$ and $48.6 \%$ were in the AAM group $(\mathrm{n}=254)$. In the AAM group, $25.2 \%(64 / 254)$ of all cows received hormone intervention $\left(79.7 \% \mathrm{PGF}_{2 \alpha}\right.$ and $20.3 \% \mathrm{GnRH}$ ). Although an effort was made to exclude any anovular cows, some cows were likely missed because $14.6 \%(37 / 254)$ of AAM cows received hormone intervention before first service. Another possibility is that the AAM threshold was set too high to create alerts for some cows with modest increases in activity during estrus, especially lame cows. Setting a threshold for alerts can be challenging because it requires a balance between false positives (if set too low) and false negatives (if set too high). Hormone interventions occurring after first service could be linked to early embryo loss, resulting in an extended length of time between estrus events in AAM cows (Santos et al., 2004). Of the AAM cows that received hormone intervention, reinsemination occurred in $92.2 \%(59 / 64)$ before the end of the 90-d study period.

\section{Time to First Service}

Cows in the AAM treatment experienced a longer time to first service than cows in the TAI treatment (hazard ratio $=0.19,95 \% \mathrm{CI}=0.15-0.24 ; P<0.01$ ) The median time to first service was $11 \mathrm{~d}$ shorter in TAI cows compared with AAM cows (Table 2).

The 11-d difference in median time to first service between treatments resulted from cows in the AAM treatment requiring natural estrus expression before the first insemination, whereas insemination of TAI cows occurred in the first $14 \mathrm{~d}$ after the end of the VWP (Figure 2a). The difference found in our study is similar to the 18 fewer days to first service Norman et al. (2009) reported in TAI managed cows compared with unsynchronized cows. The proportion of AAM cows not inseminated by 25 and $46 \mathrm{~d}$ past the VWP was 21.7 and $10.6 \%$, respectively. These missed inseminations represent anovular cows or estrus events undetected by the AAM system.

\section{Probability of Pregnancy to First AI}

Probability of pregnancy to first AI was not different between TAI and AAM treatments $(P=0.81$; Table 2 ). Cows undergoing TAI in our study were subject to presynchronization $(\mathrm{G} 7 \mathrm{G})$ before first service, which should have enhanced fertility by reducing variation in time to ovulation (Bello et al., 2006). The ability to use hormone intervention on AAM cows not displaying increased activity may have offset some of the presynchronization advantage.

Herd affected probability of pregnancy to first AI $(P<0.01$; Table 2). The differences between herds resulted from a lesser probability of pregnancy to first $\mathrm{AI}$ in herd $\mathrm{C}(30.1 \pm 3.6 \%)$ and a greater probability of pregnancy to first AI in herd A $(52.8 \pm 3.5 \%)$, whereas herd $\mathrm{B}$ fell in the middle $(41.0 \pm 4.2 \%)$. Reproductive performance before the study began was least in herd $\mathrm{C}$ (pregnancy rate $=17,16$, and $10 \%$ for herds A, B, and C, respectively, in September 2012; PCDART) and continued inferior performance was expected. Although the study design attempted to eliminate anovular cows, herd A VWP was $20 \mathrm{~d}$ longer and may have been an 


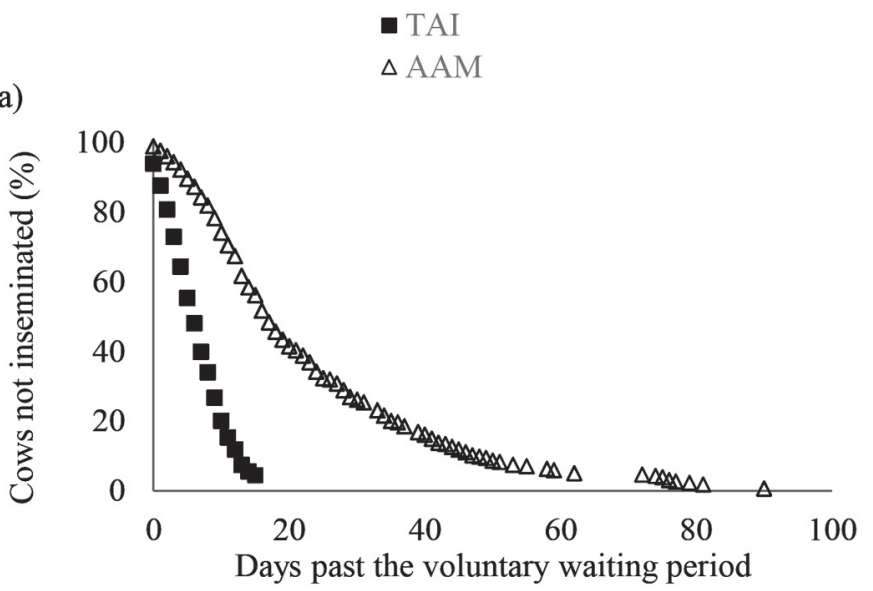

b)

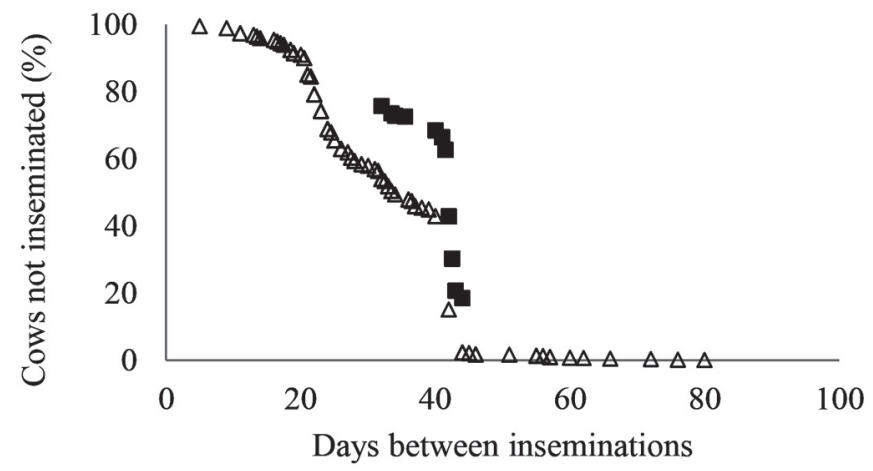

c)

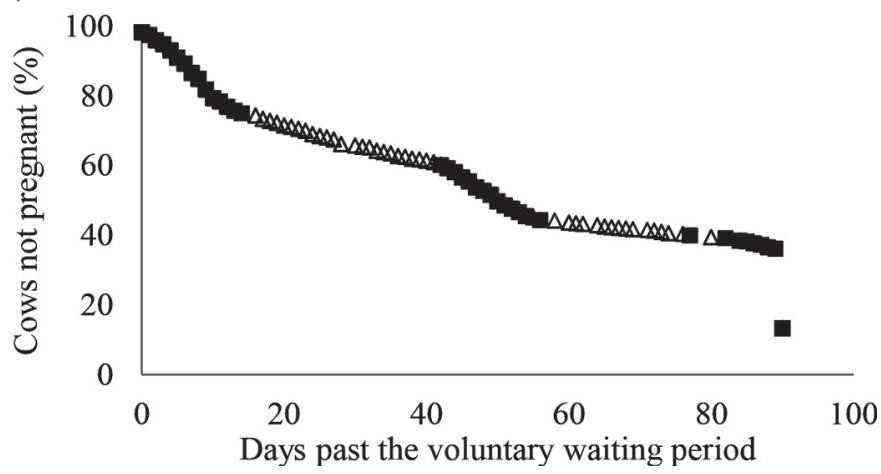

Figure 2. Survival curves representing (a) time to first service in days past the voluntary waiting period, (b) service interval, and (c) time to pregnancy in days past the voluntary waiting period for dairy cows inseminated according to timed artificial insemination (TAI) or automated activity monitoring with hormone intervention (AAM). Time to first service was longer for the AAM treatment (hazard ratio $=0.19 ; P<0.01)$ and service interval was shorter for the AAM treatment (hazard ratio $=2.23 ; P<0.01$ ). No treatment difference was observed for time to pregnancy (hazard ratio $=1.00 ; P=0.97$ ).

advantage by giving cows longer to establish normal cyclicity before consideration for insemination. Stevenson and Phatak (2005) found that delaying first service 3 wk (from 56 to 77 DIM) improved CR by $20.1 \%$. Probability of pregnancy at first AI was not affected by parity category $(P=0.61)$ or interactions between treatment and covariates.

\section{Probability of Pregnancy to Repeat AI}

Probability of pregnancy to repeat AI was not was not different between TAI and AAM treatments $(P=$ 0.88 ; Table 2). Herd did affect probability of pregnancy to repeat AI $(P<0.01$; Table 2$)$. The herd effect resulted from a lesser probability of pregnancy to repeat $\mathrm{AI}$ in herd $\mathrm{C}(31.1 \pm 4.3 \%)$ compared with herd B (54.5 $\pm 5.6 \%)$. Probability of pregnancy to repeat AI was not different between herds B and A $(40.1 \pm 5.0 \%)$. Similar to probability of pregnancy to first AI, herd C was expected to maintain an inferior performance compared with herds A and B. Probability of pregnancy to repeat AI was not affected by parity category $(P=0.43)$, BCS category $(P=0.14)$, or interactions between treatment and covariates.

\section{Service Interval}

Cows in the AAM treatment experienced a shorter service interval than cows in the TAI treatment (hazard ratio $=2.23,95 \% \mathrm{CI}=1.73-2.88 ; P<0.01)$. The median service interval for TAI cows was $17.5 \mathrm{~d}$ longer than AAM cows (Table 2).

The 17.5-d difference in median service interval resulted from the ability to rebreed open AAM cows as soon as the AAM system detected them returning to estrus. Conversely, TAI cows did not undergo reinsemination until after pregnancy diagnosis, regardless of observed estrus activity (Figure 2b). The long service interval identified in TAI cows in our study emphasizes why many herds have chosen to combine TAI and visual detection of estrus or AAM, rather than using TAI and resynchronization alone. Similarly, using hormone intervention to supplement AAM is also a common practice to deal with cows not detected in estrus using AAM alone.

\section{Pregnancy Loss}

Pregnancy loss was not different between TAI and AAM treatments $(P=0.20$, Table 2$)$. Lee and Kim (2007) also found no difference in pregnancy loss between synchronized animals (9.0\%) and nonsynchronized animals (6.3\%). The pregnancy loss observed in both treatments $(8.2-12.1 \%)$ was similar to previous reports of $7.2 \%$ pregnancy loss between 28 and $84 \mathrm{~d}$ (Silke et al., 2002) and $12.5 \%$ pregnancy loss between 31 and $45 \mathrm{~d}$ (Chebel et al., 2004). Pregnancy loss was not affected by herd $(P=0.32)$, parity category $(P=$ 
$0.15)$, BCS category $(P=0.51)$, or interactions between treatment and covariates.

\section{Time to Pregnancy}

Cows in the AAM treatment experienced a similar relative pregnancy rate to cows in the TAI treatment (hazard ratio $=1.00,95 \% \mathrm{CI}=0.84-1.18 ; P=0.97$; Table 2). Therefore, despite the 11-d longer time to first service, cows in the AAM treatment were able to catch up to those in the TAI treatment. The majority of this effect can be attributed to the 17.5-d shorter service interval in the AAM treatment.

Despite the lack of difference in relative pregnancy rate between treatments, differences in pattern of pregnancy establishment are evident (Figure 2c). The proportion of cows not pregnant in the AAM treatment decreases steadily over time, illustrating the distribution of natural estrus expression among those cows. Conversely, cows in the TAI treatment were grouped so that the synchronization protocol began every $14 \mathrm{~d}$. Therefore, the proportion of cows not pregnant in the TAI treatment decreases according to the CR over the 14-d insemination window at the end of the herd VWP. The same pattern repeats from 42 to 55 and 77 to $90 \mathrm{~d}$ past the herd VWP, indicating when resynchronization TAI occurred.

\section{Proportion of Pregnant Cows at $90 \mathrm{~d}$ Past the VWP}

The proportion of pregnant cows at $90 \mathrm{~d}$ past the VWP was not different between TAI and AAM treatments $(P=0.67$; Table 2$)$. This lack of difference is consistent with no significant difference in time to pregnancy between treatments. Herd $(P<0.01)$ affected the proportion of pregnant cows at $90 \mathrm{~d}$ past the VWP. The proportion of pregnant cows at $90 \mathrm{~d}$ past the VWP was 24\% less for herd C than either herd A or B (Table 2). The reduced probability of pregnancy to first AI observed in herd $\mathrm{C}$ support these results. The proportion of pregnant cows at $90 \mathrm{~d}$ past the VWP was not affected by parity category $(P=0.31)$ or interactions between treatment and covariates.

\section{Further Discussion}

Successful implementation of TAI as an aid or replacement to visual estrus detection has occurred on many farms. However, TAI requires strict compliance and dedicated labor for success. Based on the results of our study, a reproductive-management program supplementing AAM with hormone intervention can achieve similar effectiveness to strict TAI. However, because our study excluded around $20 \%$ of the cows in these herds because of abnormal reproductive tract examinations, low or high BCS, and metabolic disorders, interpretation of results for on-farm implementation should be performed cautiously, keeping in mind that those cows omitted from our study would be more likely to respond to TAI than AAM.

Although not evaluated in our study, a more practical on-farm practice could be to supplement AAM with TAI. Recently, Fricke et al. (2014b) evaluated the effectiveness of TAI, with or without AAM at first service. They found that supplementing TAI with AAM reduced time to first service by 7.5 to $12.4 \mathrm{~d}$, but decreased CR by $8.0 \%$ as compared with TAI alone. In another study, Stevenson et al. (2014) compared pregnancy rates of cows inseminated to a presynchronization, Ovsynch, Resynch TAI protocol with cows inseminated using AAM with hormone intervention if not inseminated by specific time points. Mean days to conception of cows in the AAM with hormone intervention group was $24 \mathrm{~d}$ less than TAI alone. Further research comparing different TAI protocols, AAM systems with various sensitivity settings, or a combination of these 2 systems may be warranted.

Although both reproductive-management strategies produced similar results in our study, the economics of each system should be analyzed before adoption on a commercial herd. The initial investment in AAM is large, but may be offset by reoccurring injection and labor costs associated with TAI. Fricke et al. (2014b) found that net present value per cow per year of TAI and AAM reproductive-management programs differed by only $\$ 4.00$ to $\$ 8.00$. This indicates that multiple reproductive-management programs can be economically feasible, depending on individual herd scenarios.

\section{ACKNOWLEDGMENTS}

The authors thank Afimilk (Kibbutz Afikim, Israel), who provided financial, software, and technical support for this study. David Corbin (Corbin Bros. Dairy, Campbellsville, KY), Larry and Doyle Embry (Longview Farms, Leitchfield, KY), Stewart and Mary Jones (Coleman Crest Dairy, Loretto, KY), and the farm staff at each of the commercial dairies are greatly appreciated for their participation in this study. Additionally, we thank Denise Ray (University of Kentucky) for her assistance with ultrasound diagnosis. We also appreciate the contributions of the herd veterinarians, associated county extension agents, and the Kentucky Dairy Development Council (Lexington, KY) consultants. 


\section{REFERENCES}

Bello, N. M., J. P. Steibel, and J. R. Pursley. 2006. Optimizing ovulation to first GnRH improved outcomes to each hormonal injection of ovsynch in lactating dairy cows. J. Dairy Sci. 89:3413-3424.

Borchers, M. R., and J. M. Bewley. 2015. An assessment of producer precision dairy farming technology use, prepurchase considerations, and usefulness. J. Dairy Sci. 98:4198-4205.

Cavalieri, J., V. Eagles, M. Ryan, and K. L. Macmillan. 2003. Comparison of four methods for detection of oestrus in dairy cows with resynchronised oestrous cycles. Aust. Vet. J. 81:422-425.

Chebel, R. C., J. E. P. Santos, J. P. Reynolds, R. L. A. Cerri, S. O. Juchem, and M. Overton. 2004. Factors affecting conception rate after artificial insemination and pregnancy loss in lactating dairy cows. Anim. Reprod. Sci. 84:239-255.

Dela Rue, B. T., C. Kamphuis, C. Burke, and J. Jago. 2014. Using activity-based monitoring systems to detect dairy cows in oestrus: A field evaluation. N. Z. Vet. J. 62:57-62.

DRMS. 2015. DairyMetrics. Dairy Records Management Systems, Raleigh, NC.

Ferguson, J. D., D. T. Galligan, and N. Thomsen. 1994. Principal descriptors of body condition score in Holstein cows. J. Dairy Sci. $77: 2695-2703$

Ferguson, J. D., and A. Skidmore. 2013. Reproductive performance in a select sample of dairy herds. J. Dairy Sci. 96:1269-1289.

Fricke, P. M., P. D. Carvalho, J. O. Giordano, A. Valenza, G. Lopes Jr., and M. C. Amundson. 2014a. Expression and detection of estrus in dairy cows: the role of new technologies. Animal 8(Suppl 1):134-143.

Fricke, P. M., J. O. Giordano, A. Valenza, G. Lopes Jr., M. C. Amundson, and P. D. Carvalho. 2014b. Reproductive performance of lactating dairy cows managed for first service using timed artificial insemination with or without detection of estrus using an activitymonitoring system. J. Dairy Sci. 97:2771-2781.

Galon, N. 2010. The use of pedometry for estrus detection in dairy cows in Isreal. J. Reprod. Dev. 56:S48-S52.

Galvão, K. N., P. Federico, A. De Vries, and G. M. Schuenemann. 2013. Economic comparison of reproductive programs for dairy herds using estrus detection, timed artificial insemination, or a combination. J. Dairy Sci. 96:2681-2693.

Heersche, G., and R. L. Nebel. 1994. Measuring efficiency and accuracy of detection of estrus. J. Dairy Sci. 77:2754-2761.

Lee, J.-I., and I.-H. Kim. 2007. Pregnancy loss in dairy cows: The contributing factors, the effects on reproductive performance and the economic impact. J. Vet. Sci. 8:283-288.

Liu, X., and S. L. Spahr. 1993. Automated electronic activity measurement for detection of estrus in dairy cattle. J. Dairy Sci. 76:29062912.

Lopez, H., L. D. Satter, and M. C. Wiltbank. 2004. Relationship between level of milk production and estrous behavior of lactating dairy cows. Anim. Reprod. Sci. 81:209-223.

López-Gatius, F., P. Santolaria, I. Mundet, and J. L. Yániz. 2005. Walking activity at estrus and subsequent fertility in dairy cows. Theriogenology 63:1419-1429.
Nebel, R. L. 2013. Attaining reproductive solutions through activity and health monitoring. Pages 75-80 in Proc. 2013 Precis. Dairy Conf. Expo, Rochester, MN. University of Minnesota, Minneapolis.

Nebel, R. L., and S. M. Jobst. 1998. Evaluation of systematic breeding programs for lactating dairy cows: A review. J. Dairy Sci. $81: 1169-1174$

Neves, R. C., and S. J. LeBlanc. 2015. Reproductive management practices and performance of Canadian dairy herds using automated activity-monitoring systems. J. Dairy Sci. 98:2801-2811.

Neves, R. C. K. E. Leslie, J. S. Walton, and S. J. LeBlanc. 2012 Reproductive performance with an automated activity monitoring system versus a synchronized breeding program. J. Dairy Sci. 95:5683-5693.

Norman, H. D., J. R. Wright, S. M. Hubbard, R. H. Miller, and J. L. Hutchison. 2009. Reproductive status of Holstein and Jersey cows in the United States. J. Dairy Sci. 92:3517-3528.

Palmer, M. A., G. Olmos, L. A. Boyle, and J. F. Mee. 2010. Estrus detection and estrus characteristics in housed and pastured Holstein-Friesian cows. Theriogenology 74:255-264.

Pursley, J. R., M. O. Mee, and M. C. Wiltbank. 1995. Synchronizatin of ovulation in dairy cows using $\mathrm{PGF}_{2 \alpha}$ and $\mathrm{GnRH}$. Theriogenology 44:915-923.

Roelofs, J. B., F. J. C. M. van Eerdenburg, N. M. Soede, and B. Kemp. 2005. Pedometer readings for estrous detection and as predictor for time of ovulation in dairy cattle. Theriogenology 64:1690-1703.

Russell, R. A., and J. M. Bewley. 2013. Characterization of Kentucky dairy producer decision-making behavior. J. Dairy Sci. 96:47514758

Saint-Dizier, M., and S. Chastant-Maillard. 2012. Towards an automated detection of oestrus in dairy cattle. Reprod. Domest. Anim. 47:1056-1061

Santos, J. E. P., W. W. Thatcher, R. C. Chebel, R. L. A. Cerri, and K. N. Galvão. 2004. The effect of embryonic death rates in cattle on the efficacy of estrus synchronization programs. Anim. Reprod. Sci. 82-83:513-535.

Senger, P. L. 1994. The estrus detection problem: New concepts, technologies, and possibilities. J. Dairy Sci. 77:2745-2753.

Silke, V., M. Diskin, D. Kenny, M. Boland, P. Dillon, J. Mee, and J. Sreenan. 2002. Extent, pattern and factors associated with late embryonic loss in dairy cows. Anim. Reprod. Sci. 71:1-12.

Stevenson, J. S., S. L. Hill, R. L. Nebel, and J. M. DeJarnette. 2014 Ovulation timing and conception risk after automated activity monitoring in lactating dairy cows. J. Dairy Sci. 97:4296-4308.

Stevenson, J. S., and A. P. Phatak. 2005. Inseminations at estrus induced by presynchronization before application of synchronized estrus and ovulation. J. Dairy Sci. 88:399-405.

Vailes, L. D., and J. H. Britt. 1990. Influence of footing surface on mounting and other sexual behaviors of estrual Holstein cows. J. Anim. Sci. 68:2333-2339. 